\title{
Fractura mandibular tardía post exodoncia de molares inferiores. Caso clínico
}

\section{Late mandibular fracture after lower third molar extraction: a case report}

\author{
A. Olivera Oliva *, M. Farnés Montpeyó*, A. Estrugo Devesa**, E. Jané Salas**, \\ C. Arranz Obispo**, A. Marí Roig****, J. López-López*****
}

\section{RESUMEN}

La extracción del tercer molar es un procedimiento común en cirugía oral. Una de las complicaciones es la fractura mandibular. Con una incidencia postoperatoria menor a un $0.005 \%$. Los factores que contribuyen al riesgo de fractura del ángulo mandibular después de una extracción del tercer molar incluyen el nivel de impactación, la anatomía del diente, infecciones locales previas, edad, sexo, presencia de formaciones quísticas y bruxismo entre otras. Presentamos un caso clínico de fractura mandibular postoperatoria a las cinco semanas tras la exodoncia del 47 y 48 incluidos en posición horizontal. El objetivo final del tratamiento de una fractura mandibular es la consolidación ósea manteniendo la oclusión dental. En nuestro caso, al no tratarse de una fractura que sobrepasaba las corticales y sin desplazamiento mandibular, no fue subsidiaria de un tratamiento quirúrgico y se resolvió con reposo funcional.

PALABRAS CLAVE: Fractura mandibular, tercer molar, complicaciones después de la extracción del tercer molar, complicación tardía, extracción.

\section{ABSTRACT}

The lower third molar extraction is a common procedure in oral surgery. One of its complications, although uncommon, is the mandibular fracture. Postoperatively, it has an incidence lower than $0.005 \%$. The factors that may contribute to the risk of the mandibular angle fracture after an extraction of the third molar include the level of impaction are the anatomy of the tooth, previous local infections, age, sex, cysts and bruxism among others. We present a clinical case of postoperative mandibular fracture at five weeks after the extraction of 47

* $\quad$ Máster en Medicina, Cirugía e Implantología Oral. Facultad de Medicina y Ciencias de la Salud (Odontología), Universidad de Barcelona.

* * Departamento de Odontoestomatología. Facultad de Medicina y Ciencias de la Salud (Odontología), Universidad de Barcelona. // Oral Health and Masticatory System Group, Institut d'Investigació Biomédica de Bellvitge (IDIBELL).

*** Departamento de Odontoestomatología. Facultad de Medicina y Ciencias de la Salud (Odontología), Universidad de Barcelona. // Oral Health and Masticatory System Group, Institut d'Investigació Biomédica de Bellvitge (IDIBELL) // Facultaitvo Especialista en Cirugía Maxilofacial, Hospital Universitario de Bellvitge

**** Jefe de Servicio de Cirugía Maxilofacial, Hospital Universitario de Bellvitge // Oral Health and Masticatory System Group, Institut d'Investigació Biomédica de Bellvitge (IDIBELL).

***** Departamento de Odontoestomatología. Facultad de Medicina y Ciencias de la Salud (Odontología), Universidad de Barcelona. // Oral Health and Masticatory System Group, Institut d'Investigació Biomédica de Bellvitge (IDIBELL) // Director Facultativo y Responsable del Área Médico Quirúrgica del Hospital Odontológico Universidad de Barcelona (HOUB). 
and 48 included in horizontal position. The ultimate treatment goal of a mandibular fracture is to reach a bone consolidation with the premorbid dental occlusion. In our case, as it was not a fracture that surpassed the cortical and there was not a mandibular displacement, it was not need a surgical treatment so it was resolved with functional rest.

KEY WORDS: Mandible fracture, third molar, complications after third molar removal, late complication, extraction.

Fecha de recepción: 24 de marzo de 2019

Fecha de aceptación: 2 de septiembre de 2019

A. Olivera Oliva, M. Farnés Montpeyó, A. Estrugo Devesa, E. Jané Salas, C. Arranz Obispo, A. Marí Roig, J. LópezLópez. Fractura mandibular tardía post exodoncia de molares inferiores. Caso clínico. 2019; 35, (3): 107-112.

\section{INTRODUCCIÓN}

Las extracciones dentales son el procedimiento más común en cirugía oral y pueden asociarse a varias complicaciones, entre las que se encuentran la osteítis alveolar, la infección secundaria, la disestesia y el sangrado. Una de las complicaciones más graves después de la extracción dental es la fractura mandibular ${ }^{(1)}$.

Los terceros molares, generalmente erupcionan entre los 17 y 26 años, pero pueden no erupcionar, hacerlo parcialmente o en su totalidad. La impactación se produce cuando no existe la erupción completa en una posición funcional normal y se establece completamente el crecimiento de la raíz. Esto puede deberse a la falta de espacio en la arcada, a la interacción con otro diente o al desarrollo en una posición anormal ${ }^{(2)}$. Un diente que está completamente impactado, puede estar completamente cubierto por tejido blando, cubierto parcialmente por hueso y tejido blando o completamente cubierto por hueso. La erupción parcial ocurre cuando el diente es visible en el arco dental pero no ha entrado en erupción en una posición funcional normal ${ }^{(2)}$.

La extracción de los terceros molares asintomáticos sigue estando en controversia. Algunas orientaciones basadas en la evidencia sugieren que la extracción profiláctica puede, en algunos casos, prevenir la posible patología asociada con la retención de éstos. No obstante, las directrices del Instituto Nacional de Excelencia en Salud y Atención (NICE), publicadas por primera vez en marzo de 2000, recomiendan un enfoque conservador. Esta guía enfatizó los riesgos asociados a la extracción de terceros molares y solicitó el fin de su eliminación profiláctica en el Servicio Nacional de Salud (NHS). En la Tabla 1 exponemos la indica-

Tabla 1. Criterios para la extracción de los terceros molares en base a

las recomendaciones de Abdulkadir et al ${ }^{(3)}$, Adam et al ${ }^{(4)}$ y Martínez JM, et al ${ }^{(5)}$.

Indicación altamente recomendable

Indicación recomendable

Actitud expectante En aquellos casos en los que los terceros molares se encuentren asintomáticos, la actitud correcta será un control clínico y radiológico acompa-

ñado de una buena higiene bucal
-Episodios infecciosos reiterados: pericoronaritis, celulitis, patología pulpar o periapical

-Caries en el tercer molar que no se pueda tratar satisfactoriamente o caries en el segundo molar intratable sin la extracción del tercer molar

-Lesión periodontal entre segundo y tercer molar

-Presencia de quistes o tumores o cuando esté involucrado en el área de extirpación de una lesión tumoral

-En pacientes que van a ser irradiados a tomar bifosfonatos u otros fármacos antiresortivos (por el alto riesgo de mucositis, osteroradionecrosis (ONR) y osteoquimionecrosis (OQN)

-En pacientes desdentados que van a soportar una prótesis removible o fija sobre el segundo molar

-Por indicación ortodóncica cuando se quiere distalar molares

-En casos de fracturas del ángulo mandibular, cuando el tercer molar está en la línea de fractura e impide la fijación

-Antes de una cirugía ortognática

- Casos de autotransplante

-Pacientes donde el tercer molar puede erupcionar con éxito y tener oclusión funcional

-Cuando la extracción suponga un riesgo a su salud o los riesgos superen los beneficios

-Impactaciones profundas sin patología local o sistémica

-Cuando el riesgo de complicaciones quirúrgicas o el riesgo de fractura por mandíbula atrófica es alto 
ción de la extracción de los terceros molares ${ }^{(3,4,5)}$.

La fractura mandibular es poco frecuente, pero es una complicación grave después de la extracción del tercer molar con una incidencia reportada entre $0,0033 \%$ y el $0,0049 \%^{(1,2,3,6)}$. Los factores de riesgos que envuelven a las fracturas mandibulares más frecuentemente descritos son: I) Edad: debido al debilitamiento de la elasticidad del hueso, estrechamiento del ligamento periodontal y aumento de la anquilosis del tercer molar al hueso. Así, por ejemplo, Libera et al. ${ }^{(7)}$ nos indican que debido a que el proceso natural de densidad ósea aumenta con la edad, en el grupo de mayor edad será necesario extraer una mayor cantidad de tejido óseo, lo que debilitará la mandíbula ${ }^{(1,8)}$.

II) Sexo masculino: por la fuerza masticatoria ${ }^{(1)}$.

III) Tipo de angulación/impactación: cuánto mayor es la profundidad de la impactación, mayor es la cantidad de tejido óseo que se debe eliminar y por lo tanto mayor susceptibilidad para la fractura ${ }^{(1,8,9)}$. Según un estudio que realizaron Ethunandan et al. ${ }^{(10)}$ las fracturas mandibulares fueron más comunes después de la extracción de los terceros molares mesioangulares, verticales, horizontales y distoangulares en orden decreciente y fueron similares a la frecuencia relativa de estas impacciones en la población general (mesioangular (45\%), vertical (40\%), horizontal (10\%) y distoangular (5\%). Por otro lado, según Andrade et al. una osteotomía extensa puede debilitar la mandíbula y hacerla más susceptible a la fractura, principalmente porque el ángulo de la mandíbula es un área de resistencia disminuida a la fractura, debido a su característica anatomía ósea y su ubicación entre la rama y el cuerpo ${ }^{(11)}$. IV) Extensión de las raíces ${ }^{(8)}$.

V) Presencia de quistes o tumores ${ }^{(8,9)}$.

VI) Enfermedades sistémicas como la osteoporosis, pueden disminuir la resistencia ósea. Las hormonas sexuales tienen efectos importantes sobre la fisiología ósea ${ }^{(8)}$.

VII) Infección preoperatoria: una infección crónica o profunda destruirá el hueso, contribuyendo así, a mayor probabilidad de fractura durante y después de la extracción del tercer molar. La pericoronaritis en el tercer molar destruye el hueso interproximal entre el $2^{\circ}$ y $3^{\circ}$ molar y habrá más probabilidad de fractura ${ }^{(8,9)}$.

El objetivo de este trabajo es presentar un caso clínico de fractura mandibular después de la extracción de un tercer molar inferior.

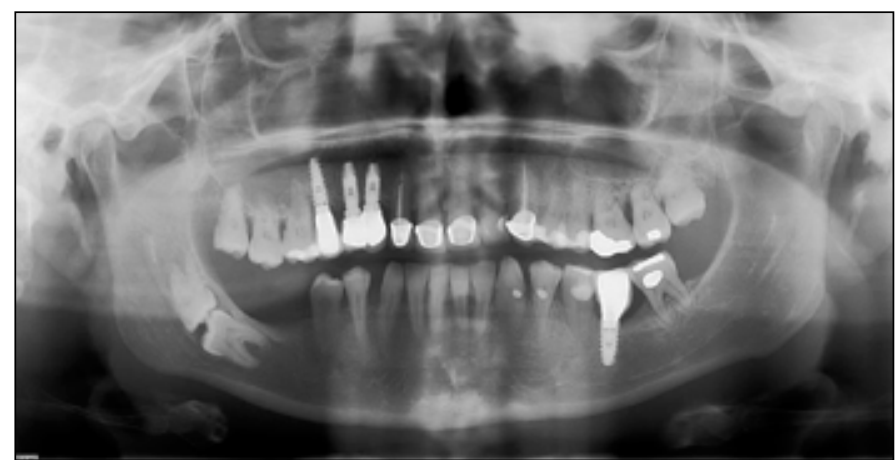

Figura 1. Radiografia, revela la inclusión horizontal del 4.7 y 4.8.

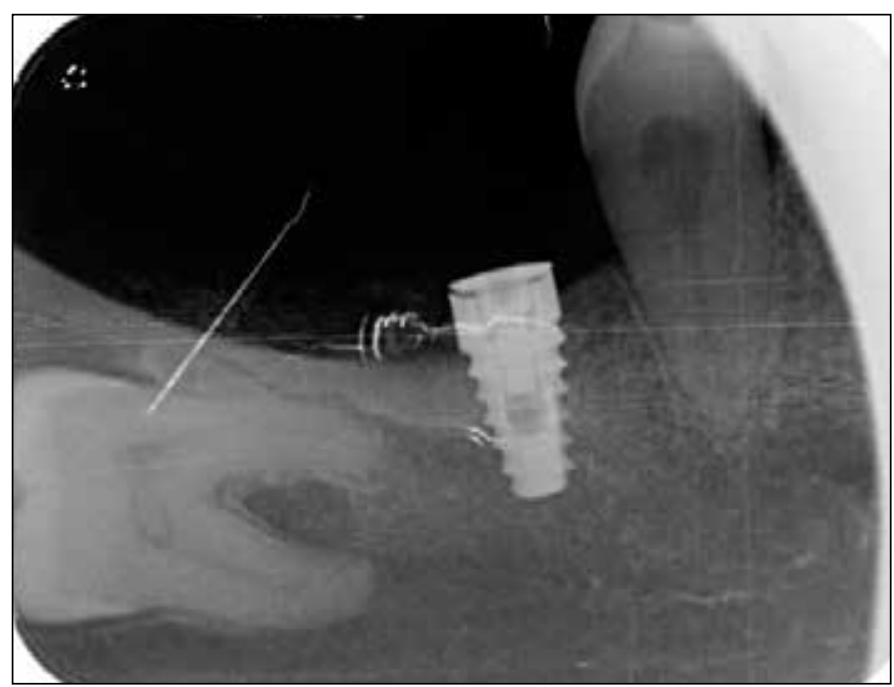

Figura 2. Radiografía periapical tras la colocación del implante en posición 4.6 .

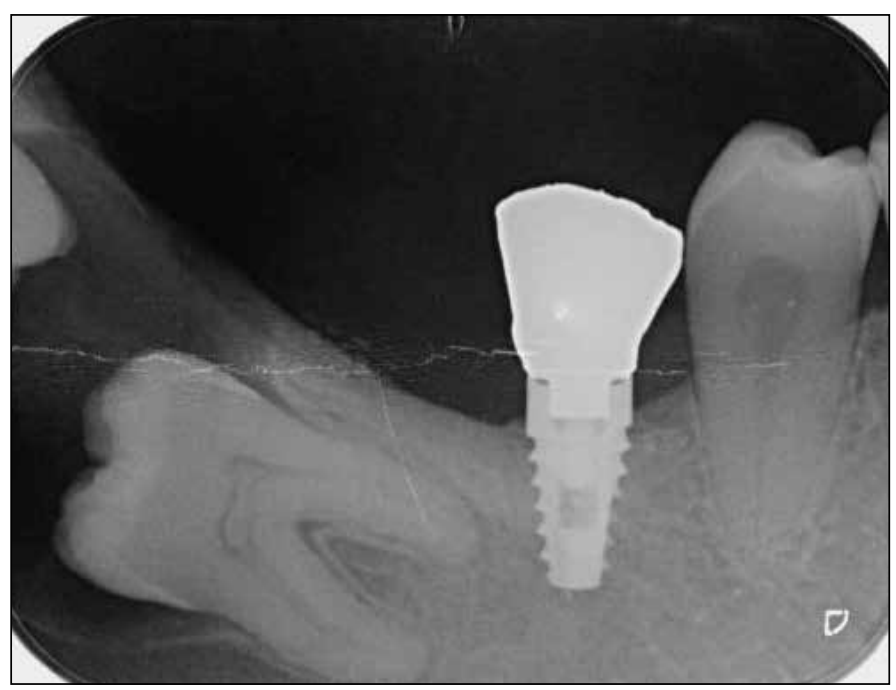

Figura 2. Radiografía periapical con imagen radiolúcida.

\section{CASO CLÍNICO}

Paciente de 54 años que acude al Hospital Odontológico Universidad de Barcelona (Máster de Me- 
dicina, Cirugía e Implantología Oral). Sin antecedentes médicos de interés, ni alergias conocidas. No presenta hábitos tóxicos. Acude para valorar la colocación de un implante en posición 4.6 y se aprecia 4.7 y 4.8 incluidos en posición horizontal. Tras valoración intraoral y radiográfica (Fig. 1) se le informa de los riesgos de la extracción de los molares incluidos y se recomienda su exodoncia. La paciente decide, a pesar de las recomendaciones, no realizar la exodoncia de dichos molares, mantener conducta expectante y colocarse el implante para reponer el diente ausente (Fig. 2). Tras 10 meses de la colocación del implante y 4 meses de la carga de éste, acude con supuración en la zona de los molares incluidos e imagen radiolúcida (Fig. 3). Se decide realizar la exodoncia de los molares y se retira la corona del 4.6 dada la cercanía al lugar operatorio para obtener una mayor visibilidad y evitar sobrecargas oclusales posteriores a la cirugía.

Tras la cirugía (que no presenta complicaciones intraoperatorias) se le indica dieta blanda, continuar con el tratamiento antibiótico, instrucciones de hi-

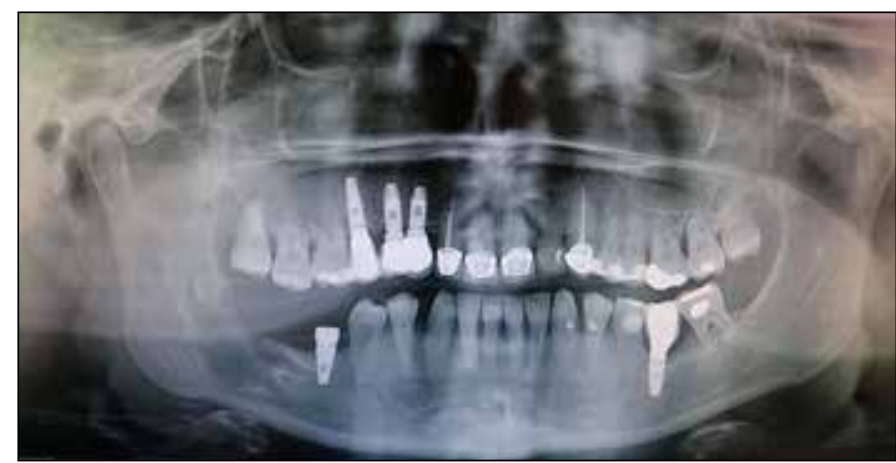

Figura 4. Radiografia panorámica tras las 5 semanas de la extracción de los molares incluidos, dónde se aprecia línea de fractura.
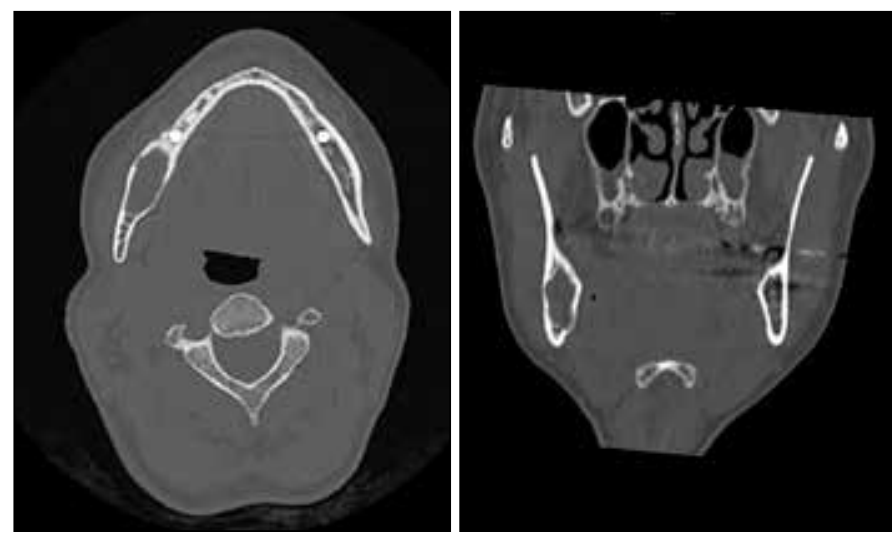

Figura 5. Corte el CBCT en el que podemos observar que la línea de fractura no sobrepasa las corticales. giene, así como terapia antiinflamatoria y analgésica. A las cinco semanas, la paciente refiere "haber sentido un ruido muy fuerte con la masticación" y pérdida de fuerza en hemimandíbula derecha. Se le realiza examen radiográfico (OPG y TC), que revela una línea de fractura que no sobrepasa las corticales y sin desplazamiento mandibular (Fig. 4,5). Dada la clínica, se le recomienda reposo funcional ya que no ha habido desplazamiento mandibular. Acude a los controles establecidos: a las cuatro semanas de la resolución radiológica y se coloca la corona del 4.6, y a las 12 semanas la paciente está asintomática y con radiología sin evidencia de patología (Fig. 6,7)

\section{DISCUSIÓN}

Si bien, la extracción quirúrgica de los terceros molares es una práctica frecuente entre los cirujanos orales, las fracturas mandibulares después de la extracción del tercer molar, es una complicación poco frecuente. Estas fracturas pueden ocurrir en el período intraoperatorio o postoperatorio. Libersa et al. ${ }^{(7)}$ en el estudio que realizaron, encontraron que la mayor incidencia de fracturas mandibulares inmediatas y tardías se asociaron a pacientes mayores de 25 años y que los hombres eran más propensos a tener fracturas mandibulares $^{(7)}$. Cuando ocurre postoperatoriamente, el mayor riesgo, según la literatura, está entre la 2 y 4 semana ${ }^{(6)}$. Según cita González et al. ${ }^{(12)}$ en su artículo, Boffano et al. ${ }^{(13)}$ remarcan que la mayoría de las fracturas $(74 \%)$ ocurren en las tres primeras semanas y el $26 \%$ ocurren durante la cirugía ${ }^{(7)}$. Para algunos autores, ocurre en este periodo de tiempo porque el tejido conectivo está sustituyendo al tejido de granulación en el alveolo postextracción ${ }^{(11)}$. Los autores inciden además en que la fractura mandibular ocurre cuando la resistencia del hueso y las fuerzas que actúan sobre ella no son iguales ${ }^{(11)}$. La reducción de la resistencia ósea puede ser causada por atrofia fisiológica, osteoporosis, procesos patológicos (lesión quística, lesión maligna, afección inflamatoria), o pueden ser secundaria a la osteotomía de la intervención quirúrgica $^{(8,11)}$.

Así pues, el factor de riesgo más importante son las fuerzas masticatorias ${ }^{(8,10)}$, debido a la posible debilitación ósea. No obstante, otros autores como Al-Belasy et al. ${ }^{(5)}$ afirmaron que la masticación no afecta la fractura mandibular tardía después de la extirpación quirúrgica de los terceros molares impactados ${ }^{(1)}$. 

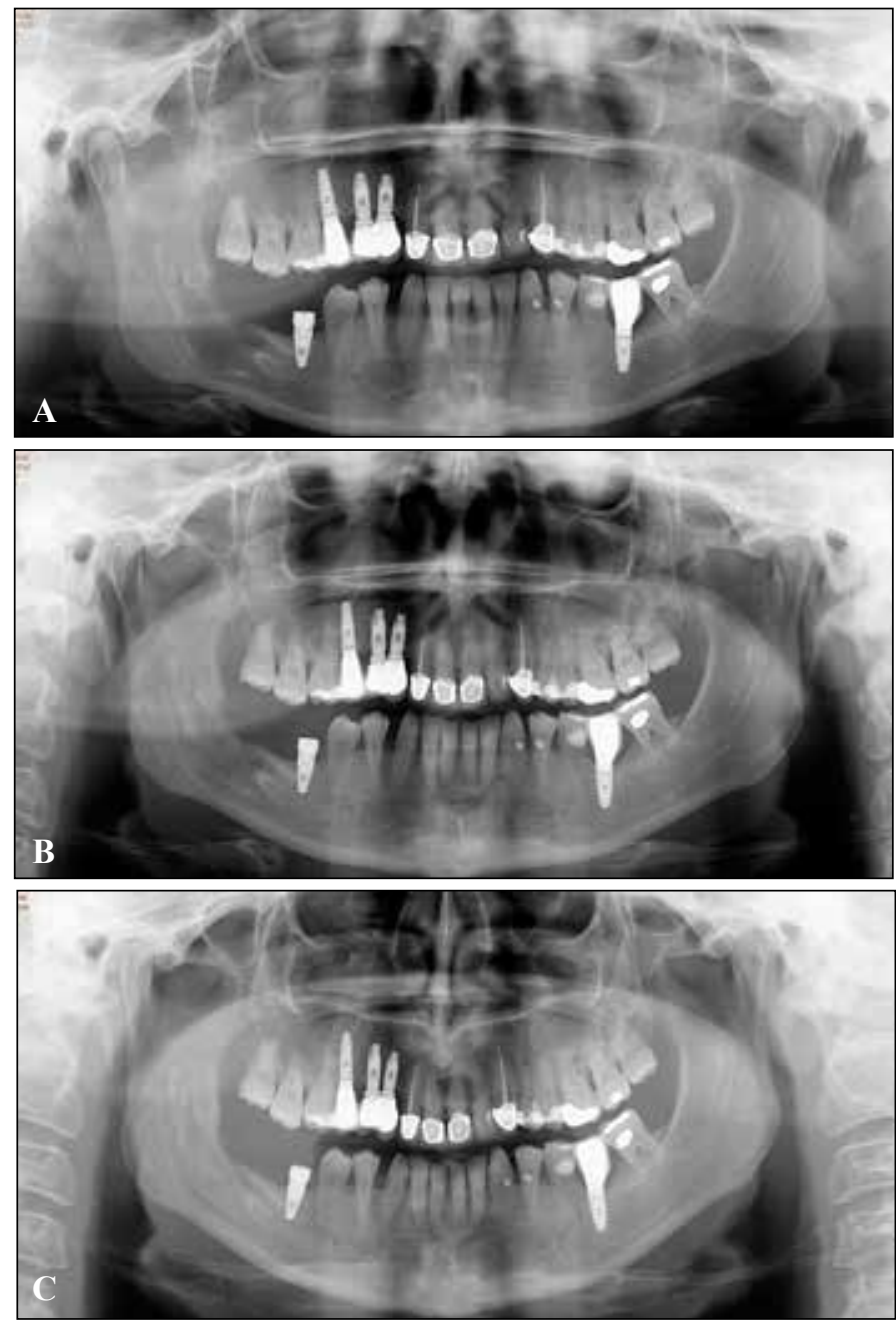

Figura 6. A) Radiografía panorámica del control del día 09-062018; B) Radiografía panorámica del control del día 17-07-2018; C) Radiografía panorámica del control del día 25-09-2018, donde se observa resolución de la fractura.

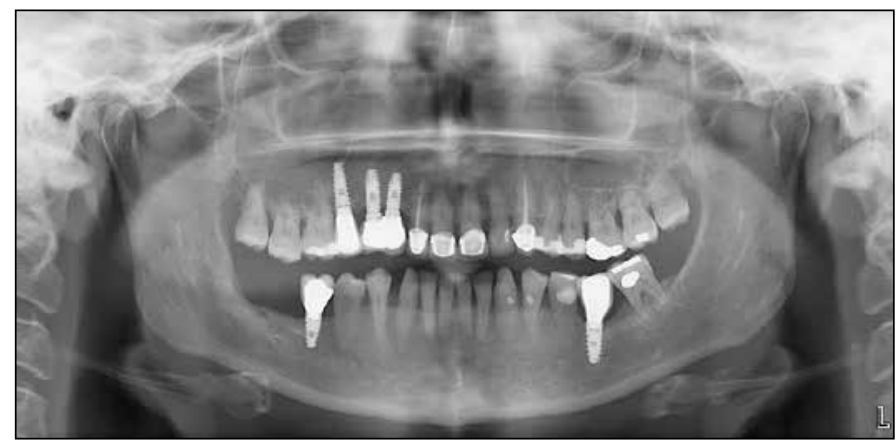

Figura 7. Radiografía panorámica de control del día 07-02-2019 dónde se observa corona del implante 4.6. colocada.

La cuestión a plantearse, es si la colocación del implante podría haber desencadenado la activación del proceso osteolítico alrededor de los molares incluidos, dado que la inflamación localizada puede acelerar dicho proceso. En nuestro caso, al hacer la incisión para realizar la colocación del implante, podría haber habido colonización de bacterias en la zona de los cordales provocando así una infección y la necesidad de exodonciar las piezas con la complicación de la fractura tardía, o bien la propia funcionalidad restablecida en la zona edéntula pudo provocar lesión mucosa, seguida de pericoronaritis e infección de los molares incluidos ${ }^{(14,15)}$.

Cuando existe una fractura mandibular, el objetivo principal del tratamiento es restablecer la oclusión dental y la función mandibular completa, ya sea con un tratamiento conservador o quirúrgico ${ }^{(6,7)}$. Solo aquellas fracturas no desplazadas, estables o incompletas en las que no se objetivan cambios en la oclusión, serán subsidiarias de ser tratadas únicamente con dieta blanda, reposo absoluto, actitud expectante y control clínico y radiológico estricto. ${ }^{(7,8)}$.

La coronectomía, descrita por primera vez en 1984 por Ecuyer y Debien, se ha evaluado principalmente en relación con la minimización del riesgo de daño al nervio dental inferior, pero, podría tener un papel importante en la disminución del riesgo de fractura mandibular asociada a molares profundamente impactados. La coronectomía u odontectomía parcial intencional, es la extirpación de la corona del tercer molar inferior dejando parte de su raíz o raíces dentro del alveolo sin tratamiento pulpar posterior, pero su indicación es dudosa y solo en algunos casos de impactación ${ }^{(16)}$.

Finalmente, es importante resaltar que el cuidado postoperatorio debe incluir una dieta blanda, evitar traumas (entre ellos deportes de contacto durante al menos cuatro semanas) e instrucciones de higiene y control periódico hasta la resolución del problema ${ }^{(9)}$.

\section{CONCLUSIÓN}

En estas situaciones, es de gran importancia que el paciente reciba una correcta explicación de los riesgos que comporta la cirugía de la extracción de cordales y que se realicen las pruebas complementarias necesarias para valorar la magnitud del riesgo y planificar la cirugía con el objetivo de reducir sus complicaciones. Una dieta blanda durante al menos 4 semanas después de la cirugía es esencial para 
prevenir la fractura del ángulo mandibular tardío. En nuestro caso, al no tratarse de una fractura mandibular completa, no precisó cirugía, únicamente reposo funcional durante 6-8 semanas.

\section{BIBLIOGRAFIA}

1. Bodner L, Brenman PA, McLeod NM. Characteristics of iatrogenic mandibular fractures associated with tooth removal: review and analysis of 189 cases. J Oral Maxillofac Surg 2011; 49: 567-72.

2. Ghaeminia H, Perry J, Nienhuijs MEL, Toedtking V, Tummers $M$. Surgical removal versus retention for the management of asymptomatic disease-free impacted wisdom teeth. Surgical removal versus retention for the management of asymptomatic disease-free impacted wisdom teeth. Cochrane Database of Systematic Reviews 2016, Issue 8. Art. N: CD003879.

3. Abdulkadir B, Mehmet A, Sirmahan C, Muhsin C, Cuneyt K. Iatrogenic mandibular fracture associated with third molar removal. Int $\mathrm{J}$ Med Sci 2011; 8: 547-53.

4. Adam N., Sandler J. NICE Guidance on the extraction of wisdom teeth-time for a Rethink?. Orthodontic Update 2018; 11: 33-5.

5. Martínez JM, Martínez N, Ortega R, Rubio LJ. Dientes Retenidos I (terceros molares). Laboratorios Normon, S.A. Madrid, 2016.

6. Al-belasy FA, Tozoglu S, Ertas U. Mastication and late mandibular fracture after surgery of impacted third molars associated with no gross pathology. J Oral Maxillofac Surg 2009; 67: 856-61.

7. Libersa P, Roze D, Cachart T, Libersa JC. Inmediate and late mandibular fractures after third molar removal. J Oral Maxillofac Surg 2002; 60: 163-5.

8. Chrcanovic B, Neto A. Considerations of mandibular angle fractures during and after surgery for removal of third molars: a review of the literature. J Oral Maxillofac Surg 2010; 14: 71-80.

9. Robert D. Complications of third molars surgery and their management. Atlas Oral Maxillofacial Surg Clin N Am 2012; 20: 233-51.
10. Ethunandan $M$, Shanahan D, Patel M. Iatrogenic mandibular fractures following removal of impacted third molars: an analysis of $130 \mathrm{ca}-$ ses. Br Dent J 2012; 24: 179-84.

11. Andrade V, De Oliveira PJ, De Moraes M, Asprino L. Late mandibular angle fracture after impacted third molar extraction- case report and review of presdisposing factors. Int $\mathrm{J}$ Odontostomat 2013; 7: 287-92.

12. González-Navarro B, Arranz-Obispo C, Albuquerque R, Jané-Salas E, López-López J. Osteomyelitis of the jaw (with pathological fracture) following extraction of an impacted wisdom tooth. A case report. J Stomatol Oral Maxillofac Surg. 2017; 118: 306-9.

13. Boffano P, Roccia F, Gallesio C, Berrone S. Pathological mandibular fractures: a review of the literatura of the last two decades. Dent Traumatol 2013; 29: 185-96.

14. Pappalardo S, Tanteri L, Brutto D, Marescalco M, Carlino V, Consolo G, Mauro M, Cappello V. Mandibular osteomyelitis due to Pseudomonas aeruginosa. Case report. Minerva Stomatol 2008; 57: 323-9.

15. Chen CP, Hertzberg M, Jiang Y, Graves DT. Interleukin-1 and tumor necrosis factor receptor signaling is not required for bacteria-induced osteoclastogenesis and bone loss but is essential for protecting the host from a mixed anaerobic infection. Am J Pathol 1999; 155: 2145-52.

16. Moreno-Vicente J, Schiavone-Mussano R, Clemente-Salas E, Marí-Roig A, Jané-Salas E, LópezLópez J. Coronectomy versus surgical removal of the lower third molars with a high risk of injury to the inferior alveolar nerve. A bibliographical review. Med Oral Patol Oral Cir Bucal 2015; 20: 508-17.

\section{AUTOR DE CORRESPONDENCIA:}

José López López

Campus Universitario de Bellvitge

Departamento de Odontoestomatología

Pabellón de Gobierno $2^{a}$ Planta

C/ Feixa Llarga s/n

08907 L'Hospitalet de Llobregat,

Barcelona (Spain)

18575jll@gmail.com

Teléfono 606457362 